\title{
Hydatid Disease of the Liver Presenting as Spontaneous Cutaneous Fistula A case report
}

Saif Ghabisha, ${ }^{1}$ "Faisal Ahmed, ${ }^{2}$ Saleh Al-Wageeh, ${ }^{1}$ Ebrahim Al-Shami, ${ }^{2}$ Khalil Al-Naggar, ${ }^{2}$ Mohammad R. Askarpour, Ali Eslahi ${ }^{3}$

\begin{abstract}
Hydatid cyst (HC) disease is a parasitic infection produced by cysts containing the Echinococcus granulosus larval phase. Patients with $\mathrm{HC}$ disease are typically asymptomatic until incidentally diagnosed or when complications occur. A rare presentation of liver $\mathrm{HC}$ is spontaneous cutaneous fistualisation. We report a 63-year-old female patient admitted in a tertiary care hospital in Ibb, Yemen, in 2019 with an infected cutaneous fistula induced by a ruptured HC. The patient underwent laparotomy and partial cystectomy with excision of the fistula tract. Upon 6-month follow-up, there was no $\mathrm{HC}$ recurrence. This report highlights the need for physicians to consider this diagnosis when faced with an unusual cutaneous fistula near organs commonly involved in HC, especially in areas where the prevalence of this disease is high.
\end{abstract}

Keywords: Complications; Cutaneous Fistula; Echinococcosis; Liver; Surgery; Case Report; Yemen.

$\mathrm{H}$ YDATID CYST (HC) IS A SERIOUS ZOONOTIC disease and a substantial health risk in endemic areas. The larval stage of the Echinococcus tapeworm causes the infection. ${ }^{1}$ Dogs are the primary host of this worm and sheep and cattle act as the intermediate hosts. Humans are accidental intermediate hosts in the parasite's dog-sheep life cycle. ${ }^{2,3}$

The most common primary infection site is the hepatobiliary system; $\mathrm{HC}$ can remain asymptomatic in the liver for a long period of time. Most early cyst detections are caused by complications of fistula formation, which are rare manifestations. ${ }^{4}$

Few adult cases of spontaneous cutaneous fistula formation due to $\mathrm{HC}$ disease have been reported. ${ }^{2,5,6}$ This case report describes a case of an adult with an infected cutaneous fistula induced by a ruptured $\mathrm{HC}$. In addition, the purpose of this report is to encourage physicians to consider this diagnosis when a patient presents with an unusual cutaneous fistula near the organs commonly involved in $\mathrm{HC}$ disease, especially in endemic areas.

\section{Case Report}

A 63-year-old female patient was admitted to a tertiary care hospital in Ibb, Yemen, in 2019 with chronic right-upper quadrant abdominal pain, which started one year ago, and a recent small skin opening located in the right eighth intercostal space medial to the anterior axillary line. She also had complaints of nausea, vomiting and fever. The patient had no history of chronic illness; however, it was noted that the patient had livestock (a sheep) living with her in her house in a village.

Upon physical examination, a mass in her right hypochondrium was found, which was tender during palpation. A $10-15 \mathrm{~mm}$ skin-opening was also noticed in this area, with a whitish membranous structure and fluid discharge [Figure 1]. Laboratory tests showed a leukocyte count of $19,000 / \mathrm{mm}^{3}$ with mild eosinophilia, an erythrocyte sedimentation rate of $100 \mathrm{~mm} / \mathrm{h}$, a haemoglobin level of $12 \mathrm{~g} / \mathrm{dL}$, total bilirubin of $2.1 \mathrm{mg} / \mathrm{dL}$ and direct bilirubin of $1.1 \mathrm{mg} /$ $\mathrm{dL}$; other liver/kidney function tests were normal. Echinococcus latex haemagglutination test and indirect haemagglutination (IHA) test were positive. Abdominal computed tomography (CT) scan revealed a $6 \times 9 \mathrm{~cm}$ irregular hypodense mass in the right lobe of the liver with some calcific areas in favour of the diagnosis of hepatic HC [Figure 2]. In support of this diagnosis, diffuse inflammatory changes were detected around the trajectory of the fistula from the abdominal cyst toward the skin. Given all findings, the patient was hospitalised based on the differential diagnosis of infected hepatic $\mathrm{HC}$ with fistualisation.

She underwent a laparotomy which revealed multiple cysts in the remnants of the right hepatic lobe, inflammatory changes in the abdominal wall and a fistula opening to the skin. Before dissecting the cyst, hypertonic saline ( $\mathrm{NaCl}: 20 \%)$ was used to wash the abdominal cavity and fistula tract to prevent any potential infection caused by contamination. All residual tissues involved in the right lobe of the liver 


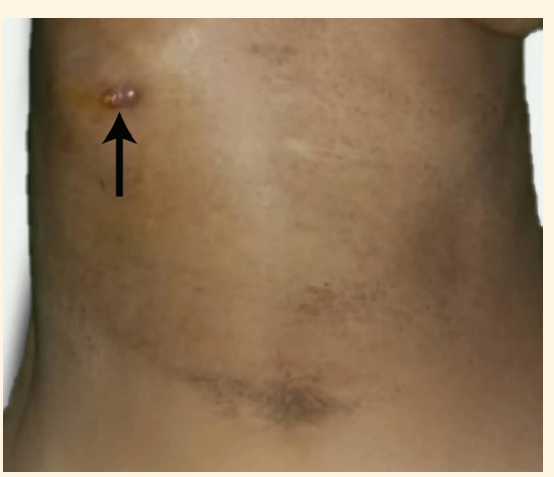

Figure 1: Photograph of the abdomen of a 63-yearold female patient showing a whitish membranous structure protruding out from the opening of a fistula (arrow).

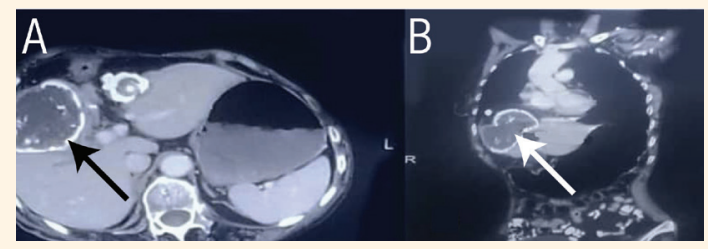

Figure 2: An abdominal computed tomography scan of a 63-year-old female patient showing the (A) axial and (B) corneal views of a calcified hydatid cyst in the right lobe of the liver (arrow).

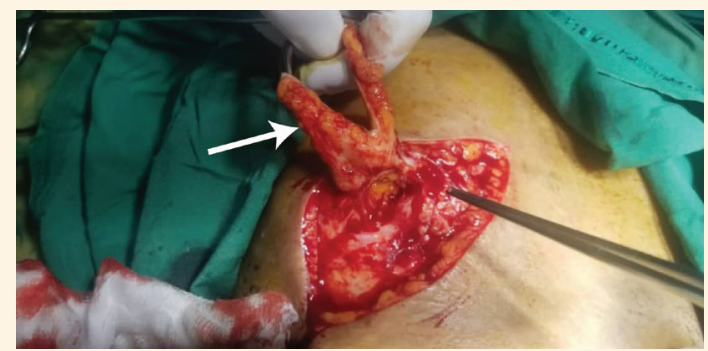

Figure 3: Intraoperative photograph showing the fistulous tract during excision (arrow).

and the cyst attached to the abdominal wall plus the fistula tract were excised [Figure 3]. Some calcification areas were observed in the cyst wall; the cyst consisted of heterogeneous degenerative contents along with numerous small daughter cysts evenly distributed in the fistula tract. Intraoperative cystic fluid examination showed protoscolices. Finally, a Foley catheter 24 Fr drain was inserted in the peritoneal cavity near the liver and the abdominal wall was reconstructed. Histopathological report of the specimen revealed a laminated cyst with many scolices with a double layer of hooklets, which is a classic presentation of Echinococcus granulosus infection. The patient had a normal post-operative course and was discharged on the 5th post-operative day on albendazole $(6 \mathrm{mg} /$ $\mathrm{kg}$ every twelve hours for four weeks) and ceftriaxone ( $1 \mathrm{~g}$ every twelve hours for one week). A follow-up abdominal CT scan, six-months post-operatively, demonstrated the absence of recurrence. Written informed consent was obtained from the patient for publication purposes and this report was approved by the local ethics committee.

\section{Discussion}

$\mathrm{HC}$ disease is a common disease in agricultural communities, including Middle Eastern countries. Cysts can rupture internally, which contaminates the biliary tract, gastrointestinal tract or the peritoneal cavity, or externally, leading to cutaneous fistulisation but this is a rare phenomenon. ${ }^{6}$ For cutaneous fistulisation to occur, the cyst must protrude into the intramuscular abdominal wall, contaminate the subcutaneous soft tissue and make a path to the surface of the skin, forming a fistula as was observed in the current patient. ${ }^{7}$ Clinical suspicion of such rare complications in endemic areas is essential for proper diagnosis when followed by proper radiological and serological investigations. ${ }^{8}$

In humans, the symptomatic phase follows an early asymptomatic infection. Types and severity of symptoms vary based on the numbers, size and location of the cyst and its pressure effect on the adjacent organs. For example, a non-complicated $\mathrm{HC}$ of the liver affects the liver locally, leading to hepatomegaly while putting pressure on the surrounding tissues to produce jaundice or abdominal pain symptoms. ${ }^{2}$ The overlying skin in patients with cutaneous fistulisation of $\mathrm{HC}$ is usually normal in appearance but rarely erythematous. ${ }^{9}$

The sign and symptoms of hepatic $\mathrm{HC}$ are not specific and they may appear in a wide range of hepatic diseases. The most common symptom is pain in the right upper quadrant or epigastric region, whereas the most frequent signs are hepatomegaly and a palpable mass. Additionally, radiological imaging methods may fail to show the direct communication between a hepatic cyst and the biliary system if hepatic $\mathrm{HC}$ is associated with a small fistula. When serological test results (including enzyme-linked immunosorbent assay, IHA test and immunoelectrophoresis) are combined with imaging findings, hydatidosis can successfully be diagnosed in $90 \%$ of cases. ${ }^{10}$

Cyst rupture into the abdominal cavity is an uncommon complication of $\mathrm{HC}$ disease. The amount of leaked material can introduce a wide range of allergic reactions from urticaria to life-threatening anaphylactic shock. Other complications include cyst communication with the biliary tree and secondary echinococcosis or symptoms caused by compression effects of the cyst on the biliary system. ${ }^{4}$ 
According to Gharbi classification, the current patient had a type $\mathrm{V}$ cyst, defined as typically inactive cysts with membrane calcification in imaging; ${ }^{11}$ however, on rare occasions, abscess formation within the calcified cyst cavity and occult abdominal trauma can lead to cutaneous fistula as was observed in the current patient. ${ }^{1,5,6}$

In the medical literature, one study reported a patient with $\mathrm{HC}$ cutaneous fistula accompanied by rib erosion. ${ }^{8}$ Typically, cysts in the right lobe of the liver invade the right lateral abdominal wall, while those in the left lobe invade the anterior abdominal wall. ${ }^{8}$ Haematogenous metastasis is an unusual cause of subcutaneous $\mathrm{HC}$ and this manifestation is mostly caused by a primary invasion of hepatic echinococcosis or contamination during previous surgery. ${ }^{12}$

Due to the rarity of $\mathrm{HC}$ and lack of prospective studies, there is no consensus on the gold-standard treatment modality. Treatment options include medical management, minimally invasive procedures and invasive surgery. For minimally invasive procedures, cysts may be aspirated percutaneously, instillated and reaspirated. In contrast, surgical options include deroofing, pericystectomy and hepatic resection..$^{5,13}$

The current patient had a complicated right-lobe liver $\mathrm{HC}$, which caused a left-lobe hypertrophy and, by intruding into the abdominal wall, led to abdominal wall injuries and cutaneous fistula formation. For this patient, a right-lobe hepatectomy was carried out, the fistula tract was excised and the abdominal wall was reconstructed.

Radical surgical approaches include lobectomy, segmentectomy and pericystectomy. Adjuvant therapy is not required before these procedures. Other conservative surgical approaches are considered less suitable to prevent disease recurrence compared to radical approaches despite having lower morbidities. ${ }^{6}$ However, if conservative surgical approaches such as cystotomy and partial cystectomy are chosen in complicated cases, it is suggested that the cystic cavity should be evacuated and the patient should receive benzimidazole treatment for 4-12 weeks to reduce the cyst size. This was not done in the current case as the patient's income was low and she could not afford it.

After resecting the skin and fistula during surgery, if fascial defects are observed in the abdominal or thoracic wall, they can be closed primarily. Even in cases of larger defects, the surgeon can use synthetic graft materials. ${ }^{14}$ In case of superinfections or abscess formation, specific antibiotics should be administered after drainage. ${ }^{15}$ In the current case, no culture test was done, therefore, the patient was discharged with empirical antibiotic therapy.

\section{Conclusion}

A cutaneous fistula, as a presentation of a ruptured $\mathrm{HC}$, is extremely rare. When drainage from the fistula does not contain the cyst material, clinical diagnosis becomes extremely difficult. Raising awareness around different $\mathrm{HC}$ complications, suggesting proper imaging modalities and serological tests are vital for accurate diagnosis of $\mathrm{HC}$, especially in endemic areas.

\section{AUTHORS' CONTRIBUTIONS}

All authors contributed to data analysis, drafting and revising the article. All authors approved the final version of the manuscript.

\section{ACKNOWLEDGEMENTS}

The authors would like to thank Shiraz University of Medical Sciences, Shiraz, Iran, as well as the Center for Development of Clinical Research of Nemazee Hospital and Dr. Nasrin Shokrpour for their editorial assistance.

\section{References}

1. Kjossev KT, Teodosiev IL. Cutaneous fistula of liver echinococcal cyst previously misdiagnosed as fistulizated rib osteomyelitis. Trop Parasitol 2013; 3:161-5. https://doi.org/10.4103/22295070.122150 .

2. Akay S, Erkan N, Yildirim M, Akay H. Development of a cutaneous fistula following hepatic cystic echinococcosis. Springerplus 2015; 4:538. https://doi.org/10.1186/s40064-015-1327-6.

3. Ghabisha S, Ahmed F, Al-Wageeh S, Al-Shami E, Al-Naggar K, Chowdhury $\mathrm{U}$, et al. Isolated giant primary splenic hydatid cyst in a 10-year-old boy: A case report. Pediatric Health Med Ther 2021; 12:55-9. https://doi.org/10.2147/PHMT.S295280.

4. Sayek I, Tirnaksiz MB, Dogan R. Cystic hydatid disease: Current trends in diagnosis and management. Surg Today 2004; 34:987-6. https://doi.org/10.1007/s00595-004-2830-5.

5. Cicero G, Blandino A, Ascenti G, D'Angelo T, Frosina L, Visalli C, et al. Superinfection of a Dead Hepatic Echinococcal Cyst with a Cutaneous Fistulization. Case Rep Radiol 2017; 2017:9393462. https://doi.org/10.1155/2017/9393462

6. Akbulut S. Parietal complication of the hydatid disease: Comprehensive literature review. Medicine (Baltimore) 2018; 97:e10671. https://doi.org/10.1097/md.0000000000010671.

7. Brunetti E, Kern P, Vuitton DA; Writing Panel for the WHOIWGE. Expert consensus for the diagnosis and treatment of cystic and alveolar echinococcosis in humans. Acta Trop 2010; 114:1-16. https://doi.org/10.1016/j.actatropica.2009.11.001.

8. Salerno S, Cracolici E, Lo Casto A. Subcutaneous rupture of hepatic hydatid cyst: CT findings. Dig Liver Dis 2006; 38:619-20. https://doi.org/10.1016/j.dld.2006.02.018.

9. Kayaalp C, Dirican A, Aydin C. Primary subcutaneous hydatid cysts: A review of 22 cases. Int J Surg 2011; 9:117-21. https:// doi.org/10.1016/j.ijsu.2010.10.009.

10. Aggarwal A, Sharma V. Primary splenic hydatidosis in a 25-yearold man: A case report. Cases J 2009; 2:8017. https://doi.org/10.4 076/1757-1626-2-8017. 
11. Lewall DB, McCorkell SJ. Rupture of echinococcal cysts: Diagnosis, classification, and clinical implications. AJR Am J Roentgenol 1986; 146:391-4. https://doi.org/10.2214/ajr.146.2.391.

12. Ambo M, Adachi K, Ohkawara A. Postoperative alveolar hydatid disease with cutaneous-subcutaneous involvement. J Dermatol 1999; 26:343-7. https://doi.org/10.1111/j.1346-8138.1999.tb03485.x.

13. Bahce ZS, Akbulut S, Aday U, Demircan F, Senol A. Cutaneous fistulization of the hydatid disease: A PRISMA-compliant systematic review. Medicine (Baltimore) 2016; 95:e4889. https:// doi.org/10.1097/md.0000000000004889.
14. Korwar V, Subhas G, Gaddikeri P, Shivaswamy BS. Hydatid disease presenting as cutaneous fistula: Review of a rare clinical presentation. Int Surg 2011; 96:69-73. https://doi.org/10.9738/ 1364.1.

15. Jayant K, Agrawal S, Agarwal R, Dayama KG. Spontaneous external fistula: The rarest presentation of hydatid cyst. BMJ Case Rep 2014; 2014:bcr2014203784. https://doi.org/10.1136/ bcr-2014-203784. 